\title{
Neural Agrin Activates a High-Affinity Receptor in C2 Muscle Cells that Is Unresponsive to Muscle Agrin
}

\author{
David C. Bowen, ${ }^{1}$ Janice Sugiyama, ${ }^{2}$ Michael Ferns, ${ }^{3}$ and Zach W. Hall ${ }^{2}$ \\ ${ }^{1}$ Regeneron Pharmaceuticals, Tarrytown, New York 10591, ${ }^{2}$ National Institute of Mental Health, National Institutes of \\ Health, Bethesda, Maryland 20892, and '3Department of Neurosurgery, Montréal General Hospital Research Institute, \\ Montréal, Québec, Canada H3G 1 A4
}

\begin{abstract}
During synaptogenesis, agrin, released by motor nerves, causes the clustering of acetylcholine receptors (AChRs) in the skeletal muscle membrane. Although muscle $\alpha$-dystroglycan has been postulated to be the receptor for the activity of agrin, previous experiments have revealed a discrepancy between the biological activity of soluble fragments of two isoforms of agrin produced by nerves and muscles, respectively, and their ability to bind $\alpha$-dystroglycan. We have determined the specificity of the signaling receptor by investigating whether muscle agrin can block the activity of neural agrin on intact $\mathrm{C} 2$ myotubes. We find that a large excess of muscle agrin failed to inhibit either the number of AChR clusters or the phosphorylation of the
\end{abstract}

Communication between skeletal muscle fibers and the motor neurons that innervate them requires the development of complex molecular machinery at the neuromuscular synapse. These events are coordinated via local signaling cues derived from the neuron, the muscle fiber, and the connecting basal lamina (Hall and Sanes, 1993). One of the earliest events in synaptic differentiation is the accumulation of acetylcholine receptors (AChRs) in the muscle membrane underlying the nerve terminal. Initially, AChRs are found along the length of the myotube surface, but when an ingrowing growth cone contacts the muscle, the AChRs become concentrated at the site of contact and extrasynaptic AChRs disappear (Salpeter et al., 1988). One factor, agrin, has been isolated from the basal lamina and is able to induce AChR clustering on cultured myotubes in the absence of innervation (McMahan, 1990; Bowe and Fallon, 1995).

Agrin exists as several isoforms, generated by alternative splicing, that differ in their biological activities and tissue localization (Ferns et al., 1992; Ruegg et al., 1992; Rupp et al., 1992; Tsim et al., 1992; Hoch et al., 1993). One of these forms is exclusively localized in neurons (Hoch et al., 1993); it contains four and eight amino acid inserts at the two most C-terminal splice sites, respectively, and is designated $\mathrm{Ag}_{4,8}$. Assays of cultured $\mathrm{C} 2$ myotubes have shown that $\mathrm{Ag}_{4,8}$ is the most active isoform in causing AChRs to cluster (Ferns et al., 1992, 1993). The predominant form in muscle, $\mathrm{Ag}_{0,0}$, which has inserts at neither of these sites, is significantly less biologically active (Ferns et al., 1992, 1993). Investigation of synapse formation in culture has shown that only

\footnotetext{
Received Jan. 10, 1996; revised March 18, 1996; accepted March 25, 1996.

This work was supported by grants from National Institutes of Health

D. C. B. and J. S, contributed equally to this manuscript.

Correspondence should be addressed to Dr. Zach W. Hall, Office of the Dircetor,

National Institute of Neurological Disorders and Stroke, National Institutes of Health, Bethesda, MD 20892.

Copyright (c) 1996 Society for Neuroscience $0270-6474 / 96 / 163791-07 \$ 05.00 / 0$
}

AChR induced by picomolar concentrations of neural agrin. These results indicate that neural, but not muscle, agrin interacts with the signaling receptor. Muscle agrin did block the binding of neural agrin to isolated $\alpha$-dystroglycan, however, suggesting either that $\alpha$-dystroglycan is not the signaling receptor or that its properties in the membrane are altered. Direct assay of the binding of muscle or neural agrin to intact myotubes revealed only low-affinity binding. We conclude that the signaling receptor for agrin is a high-affinity receptor that is highly specific for the neural form.

Key words: acetylcholine receptor; agrin; dystroglycan; muscle; receptor; synaptogenesis

neural-derived agrin is responsible for initiating AChR clustering at sites of nerve-muscle contact (Reist et al., 1992).

Despite its importance in synapse formation, the mechanism of agrin action remains unknown. Recent experiments aimed at identifying the receptor that mediates agrin-induced AChR clustering have identified $\alpha$-dystroglycan as the major agrin-binding protein in muscle (Bowe et al., 1994; Campanelli et al., 1994; Gee et al., 1994; Sugiyama et al., 1994). $\alpha$-Dystroglycan is a component of the dystrophin-glycoprotein complex that spans the muscle cell membrane and is thought to link the cytoskeleton and the extracellular matrix (Ervasti and Campbell, 1991, 1993). Although $\alpha$-dystroglycan has been postulated to be the agrin receptor responsible for AChR clustering (for review, see Bloch and Randall, 1994; Fallon and Hall, 1994; Sealock and Froehner, 1994), one troubling aspect of this hypothesis is that isolated $\alpha$-dystroglycan binds soluble fragments of both $\mathrm{Ag}_{4,8}$ and $\mathrm{Ag}_{0,0}$ with similar affinities (Sugiyama et al., 1994), even though these fragments differ by 10,000 -fold in their biological activity (Ferns et al., 1993). We report here experiments aimed at determining the specificity of the agrin signaling receptor in situ. We find that the signaling receptor seems to be highly specific, becoming activated by binding the neural, but not the muscle, form of agrin with high affinity.

\section{MATERIALS AND METHODS}

Cell culture. $\mathrm{C} 2$ cells were cultured as described previously (Gordon and Hall, 1989). Briefly, myoblasts wcre plated onto tissue culture dishes or chamber slides (Nunc, Naperville, IL) and maintained in C2 growth medium (DME-H21 conlaining 20\% fetal calf serum, 2 mm glutamine, $0.5 \%$ chick embryo extract (Gibco, Gaithersburg, MD), and penicillin/ streptomycin. At confluence, the cells were induced to differentiate by switching the medium to $\mathrm{C} 2$ fusion media (DME-H21 containing 5\% horse serum and $2 \mathrm{~mm}$ glutamine).

Recombinant agrin production. Soluble agrin was produced by transiently transfecting COS cells with rat agrin constructs containing the C-terminal half of neural $\left(\mathrm{C}-\mathrm{Ag}_{4.8}\right)$ and muscle $\left(\mathrm{C}-\mathrm{Ag}_{0,0}\right)$ agrin as de- 
scribed by Ferns et al. (1993). COS cells were transfected using an adenovirus-mediated DEAE-dextran method of transfection (Forsayeth and Garcia, 1994). The conditioned medium containing the secreted agrin was collected, and the concentration of agrin was measured by comparing the collected samples with a known amount of agrin on Western blots. Purified, soluble agrin was produced from HeLa suspension cultures infected with an agrin recombinant vaccinia virus as described previously (Sugiyama et al., 1994).

Antibodies. The rabbit serum antibody to purified rat agrin was made commercially by Babco (Richmond, CA). The antibody against rat agrin, mAb 131, was purchased from Stressgen Biotech (Victoria, BC, Canada). The antibody against the AChR $\beta$ subunit, $\mathrm{mAb} 124$, was a gift from Jon Lindstrom. ${ }^{25}$ I-labeled secondary antibodies and HRP-conjugated antibodies were purchased from Amersham (Arlington Heights, IL).

Agrin-induced AChR clustering assays. C2 myotube cultures were grown on chamber slides as described above. After $2 \mathrm{~d}$ in $\mathrm{C} 2$ fusion medium, soluble agrin (conditioned medium from agrin-transfected COS cells) was added for $18 \mathrm{hr}$ to induce $\Lambda \mathrm{ChR}$ clustering as described previously (Ferns et al., 1993). Excess muscle agrin (up to 1000-fold) was added simultaneously with the neural agrin to determine whether muscle agrin was able to inhibit neural agrin-induced clustering. Myotubes with AChR clusters were visualized by staining the cultures with rhodamine-conjugated $\alpha$-bungarotoxin for $1 \mathrm{hr}$ at $37^{\circ} \mathrm{C}$. The cultures were then fixed in $2 \%$ paraformaldehyde for $20 \mathrm{~min}$, mounted in paraphenylenediamine, and visualized under fluorescence optics. AChR clusters were quantified by counting 10 random visual fields under a rhodamine filter with a $40 \times$ objective lens.

Agrin-induced phosphorylation of the AChR $\beta$ subunit. To assay AChRtyrosine phosphorylation, $\mathrm{C} 2$ myotube cultures were treated without or with neural agrin (100 pM), muscle (10 $\mathrm{nm}$ ) agrin, or both for $1 \mathrm{hr}$. Treated cells were harvested in calcium/magnesium-free PBS containing $1 \mathrm{~mm}$ sodium orthovanadate, pelleted, and resuspended in extraction buffer (25 mM Tris, $\mathrm{pH} 7.5,25 \mathrm{~mm}$ glycine, $150 \mathrm{~mm} \mathrm{NaCl}, 5 \mathrm{~mm}$ cach EDTA and EGTA, $50 \mathrm{~mm}$ sodium fluoride, $1 \mathrm{~mm}$ sodium orthovanadate, and the protease inhibitors PMSF, benzamidine, $N$-ethylmaleimide, and sodium tetrathionate) containing $1 \%$ Triton X-100. Insoluble materials were removed by centrifugation for $4 \mathrm{~min}$. Solubilized AChRs were isolated by incubating the extracts with $\alpha$-bungarotoxin conjugated to Sepharose beads and then boiling the beads in SDS-sample buffer to elute bound proteins. The proteins were separated by SDS-PAGE and transferred to nitrocellulose membranes.

The nitrocellulose was probed with a phosphotyrosine antibody, mAb 4G10, followed by HRP-conjugated sheep anti-mouse Ig, and then visualized using the enhanced chemiluminescence method (Amersham). To identify the phosphotyrosine-immunoreactive bands and to confirm that equal amounts of $A C h R$ were present in all lanes, the blots were stripped with acid treatment $(200 \mathrm{mM}$ glycine and $0.2 \%$ Tween $20, \mathrm{pH} 2.5)$ for 20 min and reprobed with mAb 124, which recognizes the AChR $\beta$ subunit.

Membrane extract preparation and agrin overlays. Membrane extracts were prepared from $\mathrm{C} 2$ myotubes and Torpedo electric organ as described previously (Sugiyama ct al., 1994). Myotubes were harvested from $15 \mathrm{~cm}$ dishes, pelleted, resuspended in a $20 \mathrm{~mm}$ HEPES buffer containing 250 $\mathrm{mm}$ sucrose, $2 \mathrm{~mm}$ each EGTA and EDTA, $1 \mathrm{~mm}$ PMSF, and $20 \mu \mathrm{g} / \mathrm{ml}$ each leupeptin and aprotinin, followed by cell lysis in a Dounce homog enizer. After removing insoluble material by centrifugation, membranes were isolated by high-speed centrifugation at $100,000 \times g$, the membrane pellet was extracted with $25 \mathrm{~mm}$ octylglucopyranoside in phosphate buffer, and insoluble material was removed by centrifugation. Torpedo membranes were prepared as described by Ma ct al. (1993). Frozen tissuc was ground and homogenized in $50 \mathrm{~mm}$ Tris containing $400 \mathrm{~mm} \mathrm{NaCl}$. The homogenate was cleared by centrifuging twice at 6000$) \times g$, followed by centrifugation at $100,000 \times g$ to pellet membranes. Torpedo membranes were then solubilized as described for $\mathrm{C} 2$ myotubes.

Membrane proteins were separated on 6\% SDS-PAGE and transferred to nitrocellulose membranes. The membranes were first blocked with a blocking buffer (10 mM Tris, pH 7.5, $150 \mathrm{mM} \mathrm{NaCl}$, and $1 \mathrm{~mm}$ each $\mathrm{MgCl}_{2}$ and $\mathrm{CaCl}_{2}$ ) containing $10 \%$ horse serum, $5 \%$ dry milk, and $0.1 \%$ Tween 20. Blocked membranes were then incubated for $2 \mathrm{hr}$ at room temperature with ${ }^{125} \mathrm{I}$-labeled neural agrin in the presence or absence of a 100 -fold excess of either muscle $\left(\mathrm{Ag}_{0,0}\right)$ or neural $\left(\mathrm{Ag}_{4,8}\right)$ agrin. Agrinbinding proteins were visualized by autoradiography.

Agrin radioimmunoassay. C2 myotubes were grown in 12-well tissue culture dishes. The radioimmunoassay (RIA) used in these experiments was adapted from Nastuk et al. (1991). Briefly, soluble agrin was diluted with DME-H21 containing 1\% BSA and added to the myotubes for 30 min at $4^{\circ} \mathrm{C}$. This was followed by $30 \mathrm{~min}$ incubations at $4^{\circ} \mathrm{C}$ with a monoclonal antibody against rat agrin, mAb 131 (diluted 1:1000), and ${ }^{125}$ I-labeled sheep anti-mouse Ig (diluted 1:400). The cells were then extracted with $0.1 \mathrm{~N} \mathrm{NaOH}$, and agrin binding was measured by gamma counting.

\section{RESULTS}

Although soluble C-terminal fragments of neural and muscle agrin differ from each other by only 12 amino acids, there is a dramatic difference in their biological activities. The concentration required for half-maximal activity of soluble neural agrin is $\sim 50$ pm. In contrast, soluble muscle agrin gives a negligible response, even at concentrations that are 10,000-fold higher (Fig $1 a)$. These results are consistent with those previously reported (Ferns et al., 1993).

\section{Muscle agrin does not competitively inhibit neural agrin-induced AChR clustering}

The large differences in biological activities between muscle and neural agrin could reflect a large difference in affinity of the two forms of agrin for the receptor that mediates their effect on clustering activity; alternatively, the two forms could have a similar affinity for the postulated receptor but differ in their ability to activate the receptor and stimulate downstream events. To test these two possibilities, we have investigated the ability of muscle agrin to compete with the neural form of agrin. If muscle agrin has an affinity for the receptor that is comparable to that of neural agrin but fails to activate the receptor, it is, in effect, a competitive inhibitor of neural agrin and should block its activity. Alternatively, if the two have affinities that differ widely, muscle agrin should be ineffective in blocking the induction of AChR clusters by neural agrin.

We initially constructed a concentration curve measuring the ability of neural agrin to induce AChR clustering in the presence or absence of 1000-fold excess muscle agrin by increasing the amount of muscle agrin added with each concentration of neural agrin tested (Fig. 1b). At all concentrations of neural agrin tested, the addition of 1000 -fold excess muscle agrin did not affect the number of AChR clusters. The highest concentration of muscle agrin used competitively to inhibit neural agrin activity, $1500 \mathrm{pm}$, does not on its own induce AChR clustering above background (see Fig. 1a). The presence of muscle agrin also had no apparent effect on the size or shape of AChR clusters induced by neural agrin (data not shown). As a further test, we chose two concentrations of neural agrin (10 and $100 \mathrm{pm})$ in the lower range of the concentration curve and tested the effect of varying excess amounts of muscle agrin $(10 \times, 100 \times$, and $1000 \times)$ on the ability of neural agrin to induce AChR clusters (Fig. 1c). Again, an excess of muscle agrin failed to inhibit the activity of neural agrin, similar to the results obtained in Hoch et al. (1994). The results of our experiments thus show that neural agrin induces AChR clusters by activating a receptor that binds muscle agrin poorly, if at all.

\section{Muscle agrin does not inhibit neural agrin-induced phosphorylation of the AChR $\beta$ subunit}

We also tested whether muscle agrin could block downstream events induced by neural agrin. Although the signaling pathway by which agrin induces the formation of $\mathrm{AChR}$ clusters is unknown, agrin has been shown to induce phosphorylation of the $\beta$ subunit of the AChR, both in chick (Wallace et al., 1991; Qu and Huganir, 1994) and mouse muscle (Ferns et al., 1996). In C2 myotubes, agrin produces a rapid, but transient, tyrosine phosphorylation of the $\beta$ subunit (Ferns et al., 1996). Tyrosine kinase inhibitors that block AChR phosphorylation also block agrin-induced AChR 


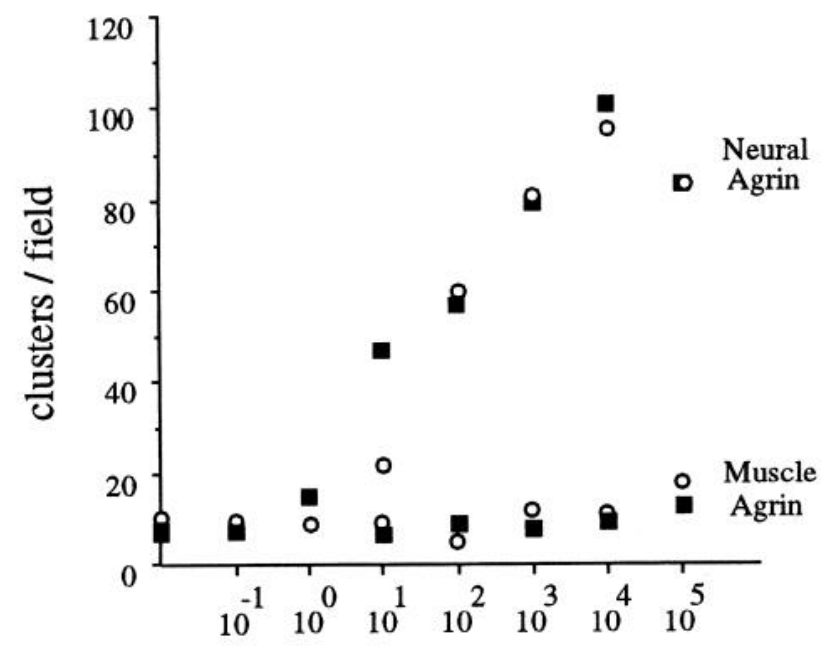

Agrin [ pM]

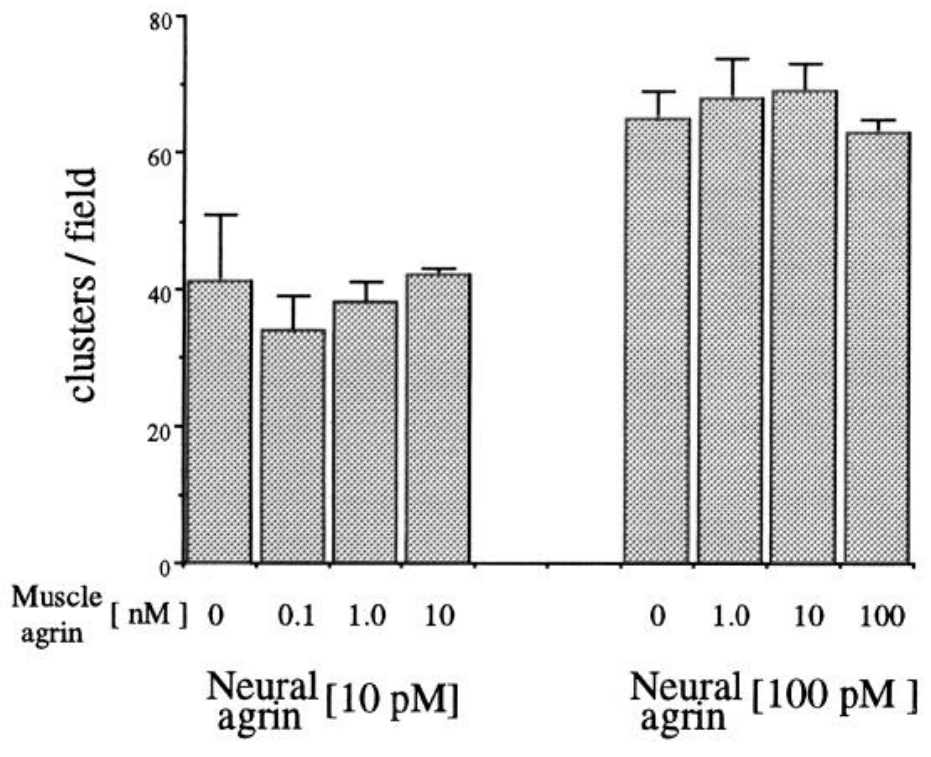

clustering under the same conditions (Wallace, 1994; Ferns et al., 1996). We tested, therefore, whether this early event in the signaling pathway for neural agrin can be blocked by muscle agrin. Neural agrin was added to myotube cultures at approximately the same concentration as that needed to induce $\mathrm{AChR}$ clustering (see Fig. 1a). When $\mathrm{C} 2$ myotubes were treated for $1 \mathrm{hr}$ (a time point that correlates to peak phosphorylation of the $\beta$ subunit) (Ferns et al., 1996) with neural agrin at $100 \mathrm{pm}$, a concentration that induces approximately half-maximal clustering, there was a strong induction of tyrosine phosphorylation of the $\beta$ subunit (Fig. 2 ). In contrast, muscle agrin failed to induce tyrosine phosphorylation of the $\beta$ subunit even at a concentration of $10 \mathrm{~nm}$. The immunoblots were reprobed with an antibody to the AChR $\beta$ subunit to confirm the identity of the phosphotyrosine band and to ensure that equal amounts of AChR were present for each sample. When a 100 -fold excess of muscle agrin was added to $100 \mathrm{pm}$ neural agrin, there was no detectable effect on the level of $\beta$-subunit phosphorylation (Fig. 2). The failure of muscle agrin to block this aspect of neural agrin signaling suggests that neural and muscle agrin differ significantly in their affinities for the signaling receptor. Thus, as with $\mathrm{AChR}$ clustering, neural agrin seems to

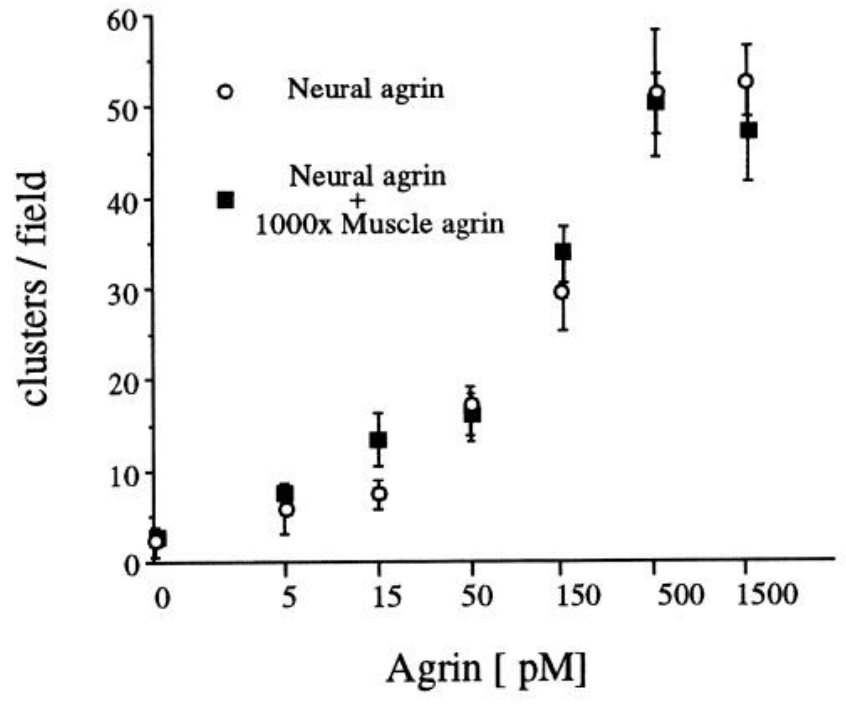

Figure 1. Excess muscle agrin does not inhibit AChR clustering induced by neural agrin. Soluble agrin was added to $C 2$ myotubes for 18 $\mathrm{hr}$, and the cultures were stained with rhodamine-conjugated $\alpha$-bugarotoxin to assay AChR clustering. Top left, Concentration curves for neural and muscle agrin. The results of two independent experiments are shown. Top right, The concentration curve for neural agrin with $(\mathbf{\square})$ and without $(O) 1000$-fold excess muscle agrin. As the neural agrin concentration was increased, the muscle agrin concentration was increased proportionally. The data for neural agrin alone are similar to that shown in top left panel. Both curves (top left, top right) were plotted on a log scale. Bottom left, Two concentrations of neural agrin (10 and $100 \mathrm{pm}$ ) plus a $10-, 100-$, or 1000 -fold excess of muscle agrin. In both experiments, an excess of muscle agrin failed to decrease the number of AChR clusters induced by neural agrin.

stimulate tyrosine phosphorylation of the $\operatorname{AChR} \beta$ subunit via a receptor that is specific for the neural isoform.

\section{Muscle agrin inhibits binding of neural agrin to $\alpha$-dystroglycan on nitrocellulose membranes}

Because $\alpha$-dystroglycan has been postulated to be the agrin receptor (for review, see Bloch and Randall, 1994; Fallon and Hall, 1994; Sealock and Froehner, 1994), we further investigated the binding of the agrin isoforms to $\alpha$-dystroglycan in vitro. If $\alpha$-dystroglycan were the receptor, a possible explanation for the discrepancy between our previous results in vitro (Sugiyama et al., 1994) and our present results is that neural and muscle agrin bind to two different, noncompeting sites on $\alpha$-dystroglycan. We therefore wished to examine competition between binding of the two isoforms to $\alpha$-dystroglycan in vitro. We extracted crude membrane preparations from Torpedo electric organ and C2 myotubes with detergent and subjected the proteins in the extract to SDS-PAGE. The separated proteins were then transferred to nitrocellulose blots and incubated with $0.5 \mathrm{nM}{ }^{125} \mathrm{I}$-labeled C-terminal, soluble neural agrin. In agreement with our previous results, a single band of 200 or $150 \mathrm{kDa}$, corresponding to $\alpha$-dystroglycan, was visual- 

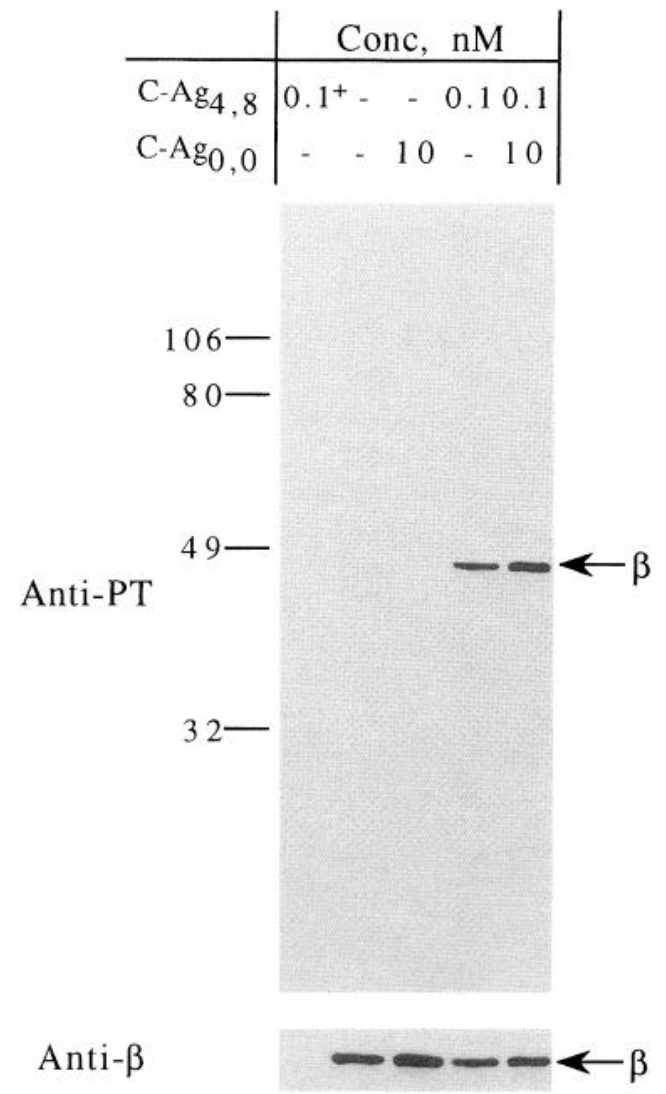

Figure 2. Muscle agrin does not competitively inhibit phosphorylation of the AChR $\beta$ subunit induced by neural agrin. $\mathrm{C} 2$ myotubes were treated with neural agrin $\left(\mathrm{C}-\mathrm{Ag}_{4,8}\right)$ for $1 \mathrm{hr}$ in the presence or absence of a 100 -fold excess of muscle agrin $\left(\mathrm{C}-\mathrm{Ag}_{0,0}\right)$. AChRs were isolated from cell extracts using $\alpha$-bugarotoxin-conjugated Sepharose beads and immunoblotted with a phosphotyrosine antibody as described in Materials and Methods. Lane 1, Blank beads ${ }^{+}$; lane 2, no agrin; lane 3, $10 \mathrm{~nm}$ muscle agrin; lane 4, $100 \mathrm{pm}$ neural agrin; lane 5, $100 \mathrm{pm}$ neural agrin $+10 \mathrm{~nm}$ muscle agrin. A 100-fold excess of muscle agrin did not alter the level of neural agrin-induced $\mathrm{AChR} \beta$ phosphorylation. The two faint tyrosinephosphorylated bands of $\sim 106 \mathrm{kDa}$ in the muscle agrin-treated extracts appeared inconsistently and were not further characterized.

ized in blots of Torpedo electric organ (Fig. $3 a$ ) and C2 muscle extracts (Fig. $3 b$ ), respectively. To determine whether muscle agrin could inhibit the binding of neural agrin to $\alpha$-dystroglycan, we added a 100 -fold excess of either unlabeled muscle or unlabeled neural agrin simultaneously with the radiolabeled neural form. In both cases, radiolabeled neural agrin binding to $\alpha$-dystroglycan in the extracts was inhibited by excess unlabeled agrin. The ability of muscle agrin to block the binding of neural agrin to $\alpha$-dystroglycan on nitrocellulose membranes indicates that muscle and neural agrin bind to isolated $\alpha$-dystroglycan in a competitive manner.

\section{Neural and muscle agrin binding to cultured myotubes}

To investigate the discrepancy between the specificity of biological activity and the nonspecificity displayed by neural and muscle agrin binding to $\alpha$-dystroglycan, we examined the binding of agrin to intact $\mathrm{C} 2$ myotube cultures. $\mathrm{C} 2$ myotubes were incubated with soluble rat agrin, and binding was measured by an RIA using mAb 131 , a mouse antibody specific for rat agrin, followed by ${ }^{125} \mathrm{I}$ labeled sheep antibody to mouse total $\mathrm{Ig}$. mAb 131 recognizes rat but not mouse agrin, thus differentiating between endogenous and exogenous agrin. Agrin binding to cultured C2 myotubes required calcium (Fig. 4a), and over the concentration range used, the half-maximal binding in different experiments ranged from 50 to $200 \mathrm{~nm}$ (Fig. $4 b$ ). The high concentrations of agrin required to see binding is in general agreement with experiments by Campanelli et al. (1994), who estimated a binding constant of 10-30 nM, based on a fluorescence immunoassay and flow cytometry of $\mathrm{C} 2$ myotubes in suspension. In contrast, Nastuk et al. (1991) estimated a value of $100-500 \mathrm{pm}$ for the binding of agrin to chick myotubes, using an RIA similar to ours.

The binding characteristics of both neural and muscle agrin isoforms to intact $\mathrm{C} 2$ myotubes indicate the presence of very abundant, low-affinity agrin-binding sites. This is in contrast to the nanomolar concentrations required to observe binding of agrin to solubilized $\alpha$-dystroglycan from C2 myotube extracts on nitrocellulose blots (Sugiyama et al., 1994) and to the picomolar concentrations required for agrin-induced AChR-clustering activity (Ferns et al., 1993). In addition, at least 1000-fold more heparin was needed to inhibit agrin binding to intact myotubes (Fig. 4c) than to inhibit agrin binding to $\alpha$-dystroglycan in vitro (Gee et al., 1994; Sugiyama et al., 1994) or to inhibit agrin-induced AChR clustering (Hirano and Kidokoro, 1989; Wallace, 1990; Saito et al., 1993). Because of these differences, it seems unlikely that the binding site that we are measuring corresponds to $\alpha$-dystroglycan or to the receptor that induces $\mathrm{AChR}$ clustering.

\section{DISCUSSION}

Understanding the mechanism by which neurally derived agrin acts requires the characterization and identification of the signaling receptor responsible for transducing information across the postsynaptic membrane. A prominent feature of the ability of agrin to stimulate AChR clustering is the remarkable specificity of the spliced forms. The addition of two inserts of four and eight amino acids, respectively, to a soluble, C-terminal fragment of agrin results in a dramatic increase in biological activity (Ferns et al., 1993). The relative activities of the cell-attached, full-length agrin forms are more difficult to estimate quantitatively but, even in this context, the neural form of agrin is clearly more active than the muscle form (Ferns et al., 1992; Ruegg et al., 1992).

The large difference between neural and muscle agrin activities raises the question of whether the two isoforms have different affinities for the physiologically active agrin receptor or whether they differ in their ability to activate downstream events. If the muscle isoform binds the signaling receptor with an affinity similar to that of neural agrin but is unable to activate the signaling pathway, it should act as an antagonist for neural agrin activity. When an excess of muscle agrin (up to 1000-fold) was added along with the neural form, however, it was unable to inhibit neural agrin-induced $\mathrm{AChR}$ clustering on $\mathrm{C} 2$ myotube cultures (Fig. $1 b, c)$ competitively. These results are consistent with those of Hoch et al. (1994), who found that a fragment of soluble muscle agrin did not inhibit the activity of the soluble neural form.

Further evidence that muscle agrin does not act as an antagonist for neural agrin comes from tyrosine phosphorylation assays. Neural agrin induces $\beta$ subunit phosphorylation at concentrations corresponding to its clustering activity (Ferns et al., 1996). Furthermore, tyrosine kinase inhibitors that prevent phosphorylation of the $\beta$ subunit also inhibit agrin-induced AChR clustering (Wallace, 1994; Ferns et al., 1996), suggesting that tyrosine phosphorylation of the $\mathrm{AChR} \beta$ subunit or other proteins is involved in the agrin-mediated clustering process. Muscle agrin, on the other 
A

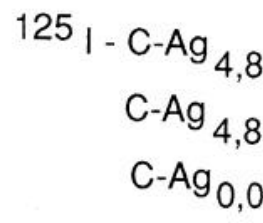

B

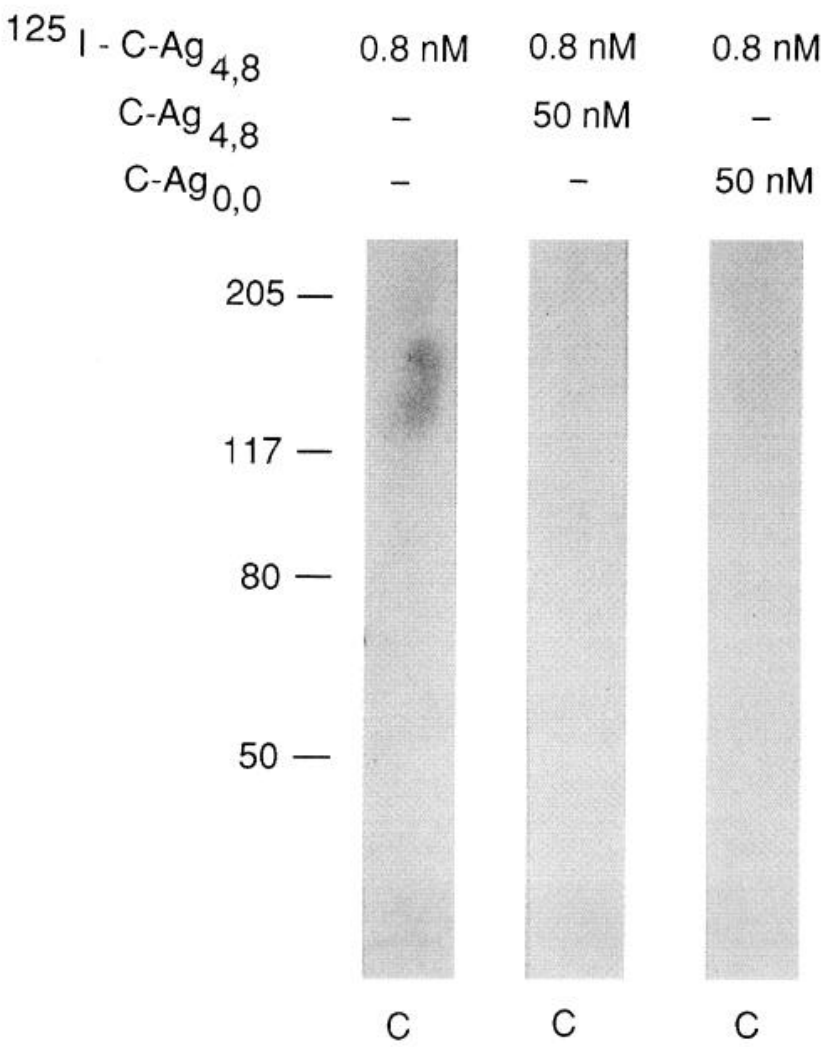

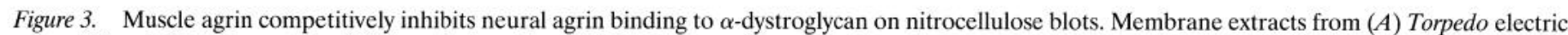

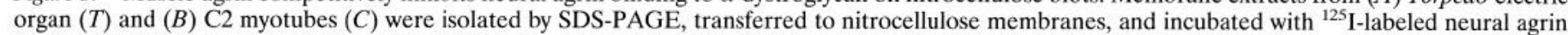

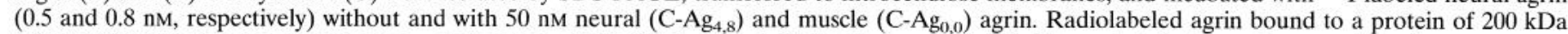
$(A)$ and $150 \mathrm{kDa}(B)$ was identified as $\alpha$-dystroglycan. This binding was inhibited by an excess of either unlabeled neural or muscle agrin.

hand, neither induces phosphorylation of the $\mathrm{AChR} \beta$ subunit at the concentrations examined (Ferns et al., 1996) nor clusters AChRs at the corresponding concentrations (Fig. 1a). When we investigated whether the presence of excess muscle agrin affects neural agrin-induced tyrosine phosphorylation of the AChR $\beta$ subunit, we found that the neural agrin retained its activity (Fig. 2). Thus, the results from experiments assaying agrin-induced AChR clustering and AChR phosphorylation show that muscle agrin does not act as a competitive inhibitor for the neural agrin signaling pathway. The differences between the two agrin isoforms must therefore arise from their differing affinities for the signaling receptor rather than through a downstream effect.

The results described above provide a strong criterion for identification of the agrin receptor or receptor complex that mediates its biological activity: it must show a much higher affinity for neural agrin than muscle agrin. Previous work has identified $\alpha$-dystroglycan as an agrin-binding, membrane-associated glycoprotein (Bowe et al., 1994; Campanelli et al., 1994; Gee et al., 1994; Sugiyama et al., 1994) and has led to the suggestion by some that it is the agrin receptor (for review, see Bloch and Randall, 1994; Fallon and Hall, 1994; Sealock and Froehner, 1994). No other agrin-binding protein in muscle or Torpedo electric organ has been identified. Some of the binding characteristics of $\alpha$-dystroglycan correlate to the characteristics of the biological activity of agrin; for example, both the binding and the AChRclustering activity are calcium-dependent and inhibited by polyanions such as heparin. Moreover, agrin binding to $\alpha$-dystroglycan is diminished in muscle cell variants that respond poorly to agrin (Campanelli et al., 1994; Gee et al., 1994; Sugiyama et al., 1994). Several features of the binding of agrin to $\alpha$-dystroglycan, however, are not consistent with its biological activity: isolated $\alpha$-dystroglycan binds both neural and muscle agrin with a binding affinity that is lower than that required for biological activity, and it shows no apparent specificity for the neural form (Sugiyama et al., 1994). We now have extended these results to show that excess muscle agrin can block the binding of neural agrin to $\alpha$-dystroglycan on nitrocellulose blots (Fig. $3 a, b$ ), thus ruling out the possibility that the two forms are binding to nonoverlapping or noninteracting sites on $\alpha$-dystroglycan.

The binding properties of $\alpha$-dystroglycan in situ could differ from those of the isolated protein, either because denaturation and refolding on the nitrocellulose blots changed its original conformation or because specific interactions with neighboring proteins in its native environment were lost. We attempted to resolve these difficulties by measuring the binding of agrin to $\alpha$-dystroglycan in intact muscle cells. Unfortunately, in $\mathrm{C} 2$ myotubes, the binding that we detected lacked both the characteristics of the agrin receptor, as defined by its biological activity, and the characteristics of binding to $\alpha$-dystroglycan, as defined on nitrocellulose blots. Agrin binding to C2 myotubes (Fig. 4b) was detectable only at much higher concentrations than those required either for binding to $\alpha$-dystroglycan on nitrocellulose blots or for biological activity, and the binding was relatively insensitive to heparin (Fig. 4c). In fact, in comparison, the binding of muscle 

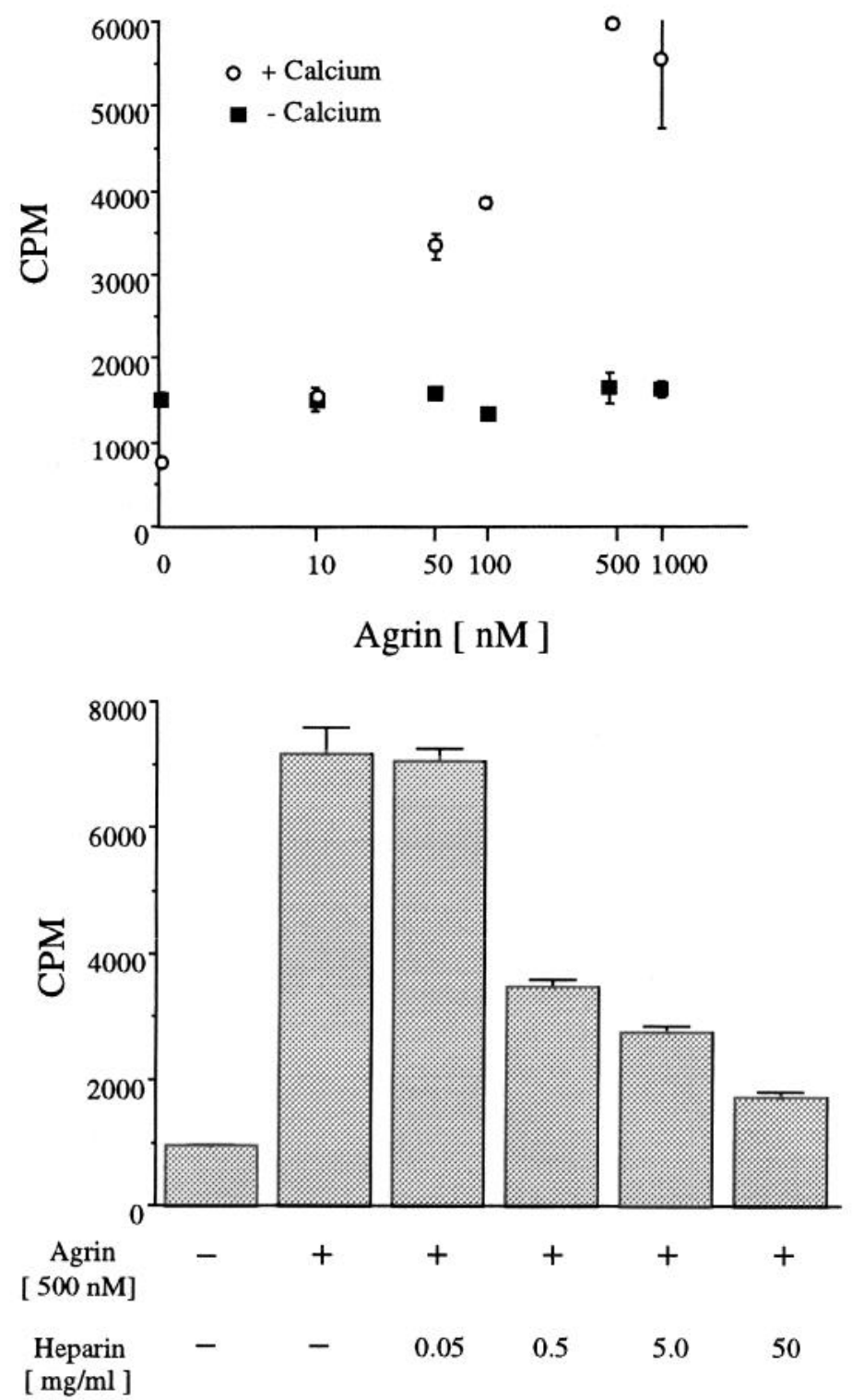

agrin seems to be higher than that of neural agrin (Fig. 4b); thus, it may also bind to a second component on $\mathrm{C} 2$ myotubes that does not detect neural agrin. Ma et al. (1993) have found a binding site in chick muscle and in Torpedo membranes, which they believe to be $\alpha$-dystroglycan, that has a higher affinity $(\sim 0.1 \mathrm{~nm})$ than that described here. The affinities of these binding sites for the two forms of agrin, however, have not been tested. In $\mathrm{C} 2$ myotubes, the high-abundance, low-affinity sites that we see may obscure the binding to $\alpha$-dystroglycan or to any other high-affinity binding site that presumably occurs. In any case, the low-affinity binding that we observe, whatever its identity, does not have the specificity of the biological response.

Because we are unable to detect the binding of agrin to $\alpha$-dystroglycan in intact myotubes, our experiments do not yield decisive information about whether it is the agrin receptor that activates the pathway leading to $\mathrm{AChR}$ clustering. In situ, $\alpha$-dystroglycan is part of a complex of six proteins associated with dystrophin and is believed to be tightly associated to one of them, the integral membrane protein $\beta$-dystroglycan (Ervasti et al., 1990; Yoshida and Ozawa, 1990; Ervasti and Campbell, 1991). This association, or others, could change the specificity of agrin

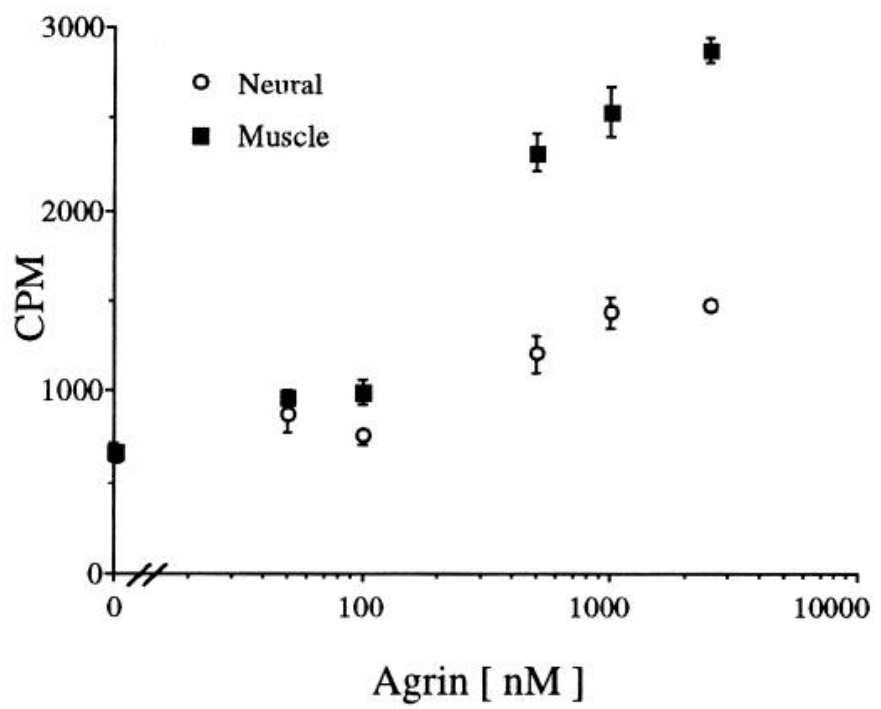

Figure 4. Binding characteristics of neural and muscle agrin to cultured $\mathrm{C} 2$ myotubes. Agrin binding to intact $\mathrm{C} 2$ myotubes was measured by RIA as described in Materials and Methods. Top left, C2 myotubes were incubated with increasing concentrations of muscle agrin in the presence $(O)$ or absence ( $)$ of calcium ( 2 mM EDTA). Top right, Neural $(\bigcirc)$ and muscle (ם) agrin-binding curves were constructed with $\mathrm{C} 2$ myotubes. Both curves (top left, top right) were plotted on a log scale. Bottom left, $\mathrm{C} 2$ myotubes were incubated with $500 \mu \mathrm{M}$ muscle agrin and increasing concentrations of heparin. The low-affinity agrin binding to intact myotubes is dependent on the presence of calcium and is relatively insensitive to heparin.

binding exhibited by $\alpha$-dystroglycan. Such a change must be a large one, because the isolated protein exhibits roughly equal affinities for the two forms of agrin, whereas the biological receptor shows a preference of over 1000 -fold for the neural form, which differs from the muscle form by only 12 amino acids. Although the role of $\alpha$-dystroglycan thus remains unsettled, the high degree of specificity that we demonstrate for the agrinsignaling receptor in situ provides an important criterion for its ultimate identification.

\section{REFERENCES}

Bloch RJ, Randall WR (1994) Up the junction. Curr Biol 4:936-938. Bowe MA, Fallon JR (1995) The role of agrin in synapse formation. Annu Rev Neurosci 18:443-462.

Bowe MA, Deyst KA, Leszyk JD, Fallon JR (1994) Identification and purification of an agrin receptor from Torpedo postsynaptic membranes: a heteromeric complex related to the dystroglycans. Neuron 12:1173-1180.

Campanelli JT, Roberds SL, Campbell KP, Scheller RH (1994) A role for dystrophin-associated glycoproteins and utrophin in agrin-induced acetylcholine receptor clustering. Cell 77:663-674.

Ervasti JM, Campbell KP (1991) Membrane organization of the dystrophin-glycoprotein complex. Cell 66:1121-1131. 
Ervasti JM, Campbell KP (1993) A role for the dystrophin-glycoprotein complex as a transmembrane linker between laminin and actin. $\mathrm{J}$ Cel Biol 122:809 - 823 .

Ervasti JM, Ohlendieck K, Kahl SD, Gaver MG, Campbell KP (1990) Deficiency of a glycoprotein component of the dystrophin complex in dystrophic musclc. Naturc 345:315-319.

Fallon JR, Hall ZW (1994) Building synapses: agrin and dystroglycan stick together. Trends Neurosci 17:469-473.

Ferns M, Hoch W, Campanelli JT, Rupp F, Hall ZW, Scheller RH (1992) RNA splicing regulates agrin-mediated acetylcholine receptor clustering activity on cultured myotubes. Neuron 8:1079-1086.

Ferns MJ, Campanelli JT, Hoch W, Scheller RH, Hall ZW (1993) The ability of agrin to cluster AChRs depends on alternative splicing and on cell surface proteoglycans. Neuron 11:491-502.

Ferns MJ, Deiner M, Hall ZW (1996) Agrin-induced acetylcholine receptor clustering in mammalian muscle requires tyrosine phosphorylation. J Cell Biol 132:937-944.

Forsayeth JR, Garcia PD (1994) Adenovirus-mediated transfection of cultured cells. Biotechniques 17:354-356.

Gee SH, Montanaro F, Lindenbaum MI, Carbonetto S (1994) The dystrophin-associated glycoprotein, $\alpha$-dystroglycan, is a functional agrin receptor. Cell 77:675-686.

Gordon H, Hall ZW (1989) Glycosaminoglycan variants in the C2 muscle cell line. Dev Biol 135:1-11.

Hall ZW, Sanes JR (1993) Synaptic structure and development: the neuromuscular junction. Neuron [Suppl] 10:99-121.

Hirano Y, Kidokoro Y (1989) Heparin and heparan sulphate partially inhibit induction of acetylcholine receptor accumulation by nerve in Xenopus culture. J Neurosci 9:1555-1561.

Hoch W, Ferns M, Campanelli JT, Hall ZW, Scheller RH (1993) Developmental regulation of highly active alternatively spliced forms of agrin. Neuron 11:479-490.

Hoch W, Campanelli JT, Harrison S, Scheller RH (1994) Structural domains of agrin required for clustering of nicotinic acetylcholine receptors. EMBO J 13:2814-2821.

Ma J, Nastuk MA, McKechnie BA, Fallon JR (1993) The agrin receptor. $\mathrm{J}$ Biol Chem 268:25108-25117.

McMahan UJ (1990) The agrin hypothesis. In: Cold Spring Harbor symposia on quantitative biology, pp 407-418. Cold Spring Harbor, NY: Cold Spring Harbor Laboratory.
Nastuk MA, Lieth E, Ma J, Cardasis CA, Moynihan EB, McKechnie BA Fallon JR (1991) The putative agrin receptor binds ligand in a calciumdependent manner and aggregates during agrin-induced acetylcholine receptor clustering. Neuron 7:807-818.

Qu Z, Huganir RL (1994) Comparison of innervation and agrin-induced tyrosinc phosphorylation of the nicotinic acctylcholine receptor. $J$ Neurosci 14:6834-6841.

Reist NE, Werle MJ, McMahan UJ (1992) Agrin released by motor neurons induces the aggregation of $\mathrm{AChRs}$ at neuromuscular junctions. Neuron 8:865-868.

Ruegg MA, Tsim KWK, Horton SE, Kroger S, Escher G, Gensch EM, McMahan UJ (1992) The agrin gene codes for a family of basal lamina proteins that differ in function and distribution. Neuron 8:691-699.

Rupp F, Ozcelik TH, Linial M, Peterson K, Francke U, Scheller R (1992) Structure and chromosomal localization of the mammalian agrin gene. J Neurosci 12:3535-3544.

Saito M, Nguyen J, Kidokoro Y (1993) Inhibition of nerve- and agrininduced acetylcholine receptor clustering on Xenopus muscle cells in culture. Dev Brain Res 71:9-17.

Salpeter MM, Marchaterre M, Harris R (1988) Distribution of extrajunctional acetylcholine receptors on a vertebrate muscle: evaluated by using a scanning electron microscope autoradiographic procedure. J Cell Biol 106:2087-2093.

Sealock R, Froehner SC (1994) Dystrophin-associated proteins and synapse formation: is $\alpha$-dystroglycan the agrin receptor? Cell 77:617-619.

Sugiyama J, Bowen D, Hall ZW (1994) Dystroglycan binds nerve and muscle agrin. Neuron 13:103-115.

Tsim KWK, Ruegg MA, Escher G, Kroger S, McMahan UJ (1992) cDNA that encodes active agrin. Neuron 8:677-689.

Wallace BG (1990) Inhibition of agrin-induced acetylcholine-receptor aggregation by heparin, heparan sulphate, and other polyanions. J Neurosci $10: 3576-3582$

Wallace BG (1994) Staurosporine inhibits agrin-induced acetylcholine receptor phosphorylation and aggregation. J Cell Biol 125:661-668.

Wallace BG, Qu Z, Huganir RL (1991) Agrin induces phosphorylation of the nicotinic acetylcholine receptor. Neuron 6:869-878.

Yoshida M, Ozawa E (1990) Glycoprotein complex anchoring dystrophin to sarcolemma. J Biochem (Tokyo) 108:748-752. 